\title{
A simple method to determine the elimination half-life of drugs displaying noncumulative toxicity
}

\author{
Deepesh Nagarajan ${ }^{1}$, Preetham Venkatesh ${ }^{2}$, Chandrani Thakur ${ }^{1}$, Akshay Datey $^{3}$, \\ Nagasuma Chandra ${ }^{1,4^{*}}$, and Dipshikha Chakravortty ${ }^{3,4^{* *}}$
}

\author{
${ }^{1}$ Department of Biochemistry, Indian Institute of Science, Bangalore - 560012. \\ ${ }^{2}$ Undergraduate program, Indian Institute of Science, Bangalore - 560012, India \\ ${ }^{3}$ Department of Microbiology and Cell Biology, Indian Institute of Science, Bangalore - 560012, India \\ ${ }^{4}$ Centre for Biosystems Science and Engineering, Indian Institute of Science, Bangalore - 560012, India \\ *nchandra@iisc.ac.in \\ **apa@iisc.ac.in
}

\section{ABSTRACT}

The pharmacokinetic characterization of a drug, especially the determination of its biological half-life, is an essential step during the early phases of drug development. An adequate half-life is amongst the many properties needed for selecting a drug candidate for clinical trials. Conversely, drug candidates possessing inadequate half-lives may be modified or eliminated from the drug discovery pipeline altogether. Several methods exist for determining the half-lives of drugs, namely HPLC, fluorescence assays, radioassays, radioimmunoassays, and elemental mass spectrometric assays. However, all these techniques are resource and labor-intensive, and cannot be used for the high-throughput half-life determination of hundreds of drug candidates. Here, we describe $\mathrm{TOX}_{H L}$ : a simple technique to determine the half-lives of compounds displaying noncumulative toxicity. To calculate the half life, $\mathrm{TOX}_{H L}$ only relies on the survival outcomes of three experiments performed on an animal model: an acute toxicity experiment, a cumulative toxicity experiment, and a multi-dose experiment at different dosing intervals. As a proof of concept, we use $\mathrm{TOX}_{H L}$ to determine the peritoneal half-life of $\Omega 76$, an antimicrobial peptide. The half-life of $\Omega 76$ determined by $\mathrm{TOX}_{H L}$ is in good agreement with results from a standard mass spectrometric method, validating this approach.

\section{Introduction}

The biological half-life of a drug is the time required for half the drug to be eliminated from an organism, provided the rate of removal can be modeled as an exponential function ${ }^{1}$. Typically, the kidneys eliminate hydrophilic drugs from circulation, while the liver acts on hydrophobic compounds, adding polar groups for later renal excretion ${ }^{2}$. Drugs undergoing hepatic metabolism may also enter bile and be excreted through feces ${ }^{3}$. Proteolysis, the degradation of proteins into smaller peptides or amino acids, is also an important process for the elimination of therapeutic peptides ${ }^{4}$. Secondary drug removal also occurs through respiration, through the salivary glands ${ }^{5}$, mammary glands ${ }^{6}$, lacrimal glands ${ }^{7}$, skin $^{8}$, and hair ${ }^{9}$.

A successful drug candidate should demonstrate adequate bioavailability ${ }^{10}$. The drug should remain at its site of action at a sufficient concentration, and for a sufficient time, for therapeutic effects to occur. During the early phases of drug development, measuring the half-life of a drug candidate in rodents is usually performed. Drug candidates possessing an adequate half-life for therapeutic effects to occur can enter later phases of development. Drug candidates possessing an insufficient half-life may need to be chemically altered and retested before entering later phases of development, or discarded altogether.

Numerous techniques exist for determining the concentrations and half-life of drugs. High performance liquid chromatography (HPLC) has been used to separate and characterize numerous small-molecule drugs based on molecular weights ${ }^{11,12}$. Fluorescence assays can track a drug based on innate fluorescence or that of an attached fluorophore ${ }^{13,14}$. Radioisotopes (typically ${ }^{14} \mathrm{C}$, but also ${ }^{32} \mathrm{P},{ }^{34} \mathrm{~S}$, etc.) incorporated into drug molecules allow them to be systemically tracked via scintillation counting ${ }^{15}$. Radioimmunoassays are competitive inhibition techniques where an unlabelled drug competes for antibody binding with its radioactive counterpart (typically labeled with ${ }^{3} \mathrm{H},{ }^{75} \mathrm{Se},{ }^{125} \mathrm{I}$, etc.) ${ }^{16-18}$. Labeling a drug with a biologically rare element (such as $\mathrm{Se}$ ) and tracking it using elemental mass spectrometry is also possible ${ }^{19}$. However, all these methods are labor-intensive, 
require sophisticated instruments, involve complicated chemical syntheses, or require handling radioisotopes. Such techniques are therefore impractical for the high-throughput screening of half-lives (100+ drugs at a time).

Here, we describe $\mathrm{TOX}_{H L}$ : a simple method for the determination of the half-lives of drugs based on survival outcomes alone. TOX $_{H L}$ involves 3 experiments: an acute toxicity experiment, a cumulative toxicity experiment, and a multi-dose experiment at different dosing intervals. Data obtained from these 3 experiments was sufficient to calculate the peritoneal half-life of an antimicrobial peptide $\left(\Omega 76^{19}\right)$ in BALB/c mice. The half-life of $\Omega 76$ calculated here was in good agreement with previous pharmacodynamic data obtained using mass spectrometry.

\section{Results}

\subsection{Defining and determining (non)cumulative toxicity}

Here, we define and contrast the terms acute toxicity, maximum nonlethal dose, cumulative toxicity, and noncumulative toxicity. We use two antimicrobial peptides: $\Omega 76^{19}$ and Pexiganan ${ }^{20,21}$, to demonstrate these concepts. $\Omega 76$ is a designed antimicrobial peptide effective against systemic infections of resistant $A$. baumannii. Here, $\Omega 76$ is used as an example drug that displays noncumulative toxicity and is compatible with $\mathrm{TOX}_{H L}$. Pexiganan is a broad-spectrum peptide that is effective at treating infected wounds. Here, Pexiganan is used as an example drug that displays cumulative toxicity and is incompatible with TOX $_{H L}$. It should be noted that the toxicity results for Pexiganan described here should not be used to assess its suitability as a topical agent, a role where systemic toxicity is unimportant.

Acute toxicity comprises the lethal effects of a single dose of a drug, rather than the combined lethal effects of multiple doses of a drug. BALB/c mice (6-8 weeks, $20 \mathrm{~g}$ ) were injected intraperitoneally with varying doses of $\Omega 76$ and Pexiganan, at 2-fold concentration increments. 5 mice per concentration cohort were used. All mice were observed for 5 days for mortality. Mice treated with both $\Omega 76$ and Pexiganan display mortality that increases in a dose-dependent manner. These results are depicted in Figure 1A. The $\mathrm{LD}_{50}$ values for $\Omega 76$ and Pexiganan were determined to be $170 \mathrm{mg} / \mathrm{kg}$ and $60 \mathrm{mg} / \mathrm{kg}$ respectively ${ }^{19}$, using linear (non-logarithmic) interpolation. However, for $\mathrm{TOX}_{H L}$, determining the maximum nonlethal dose (MND) rather than the $\mathrm{LD}_{50}$ is more important. We define the MND as the highest concentration of a drug, administered as a single intraperitoneal dose, that does not cause any mortality in mice observed for 5 days. Here, the MND values for $\Omega 76$ and Pexiganan are 32 $\mathrm{mg} / \mathrm{kg}$ and $64 \mathrm{mg} / \mathrm{kg}$ respectively (Figure $1 \mathrm{~A}$, shown in arrows). These results are consistent with our previously reported values, which were performed on smaller cohorts ${ }^{19}$.

Cumulative toxicity comprises the lethal effects of multiple doses of drug when injected intraperitoneally at MND concentrations. $10 \mathrm{BALB} / \mathrm{c}$ mice (6-8 weeks, $20 \mathrm{~g}$ ) were treated with 11 doses of Pexiganan, administered at the MND (32 mg/kg) at 12 hour intervals, over the course of 5 days. Despite Pexiganan never exceeding the MND concentration, $100 \%$ mortality was observed. This indicates that a single dose of Pexiganan causes a small amount of damage in mice, which is insufficient to cause mortality. However, this damage is not reversed within the dosing interval timespan (12 hours), and additional doses only serve to incrementally increase the total damage caused to mice, until a threshold is reached and death occurs. Autopsies indicate that the mechanism of cumulative toxicity for Pexiganan may involve cumulative damage to peritoneal organs culminating in intestinal inflammation (Figure 2B), which is absent in mice treated with $\Omega 76$ (Figure 2A).

Noncumulative toxicity can be defined in contrast to cumulative toxicity. For a drug to display noncumulative toxicity, multiple doses injected intraperitoneally at MND concentrations should cause no mortality. 10 BALB/c mice (6-8 weeks, 20 g) were treated with 11 doses of $\Omega 76$, administered at the $\mathrm{MND}(64 \mathrm{mg} / \mathrm{kg})$ at 12 hour intervals, over the course of 5 days. $\Omega 76$ never exceeded the MND concentration, and consequently neither intestinal inflammation nor mortality was observed (2A, Figure 1B). Our previous work ${ }^{19}$ further confirmed that $\Omega 76$ does not cause nephrotoxicity or hepatotoxicity in similarly treated mice. $\mathrm{TOX}_{H L}$ can only be used for drugs displaying noncumulative toxicity, which makes performing the acute and (non)cumulative toxicity experiments depicted in Figure 1A,B essential.

\subsection{Experiments and input terms required for $\operatorname{TOX}_{H L}$}

Once noncumulative toxicity for a given drug is established (Figure 1B), $\mathrm{TOX}_{H L}$ relies on the results of 2 experiments: acute toxicity experiments (Figure 1A) and multi-dose toxicity experiments (Figure 1C).

Multi-dose toxicity experiments are required to determine $\Delta \mathrm{t}_{M}$ : the dosing interval that causes mortality, and $S_{\Delta t_{M}}:$ the 

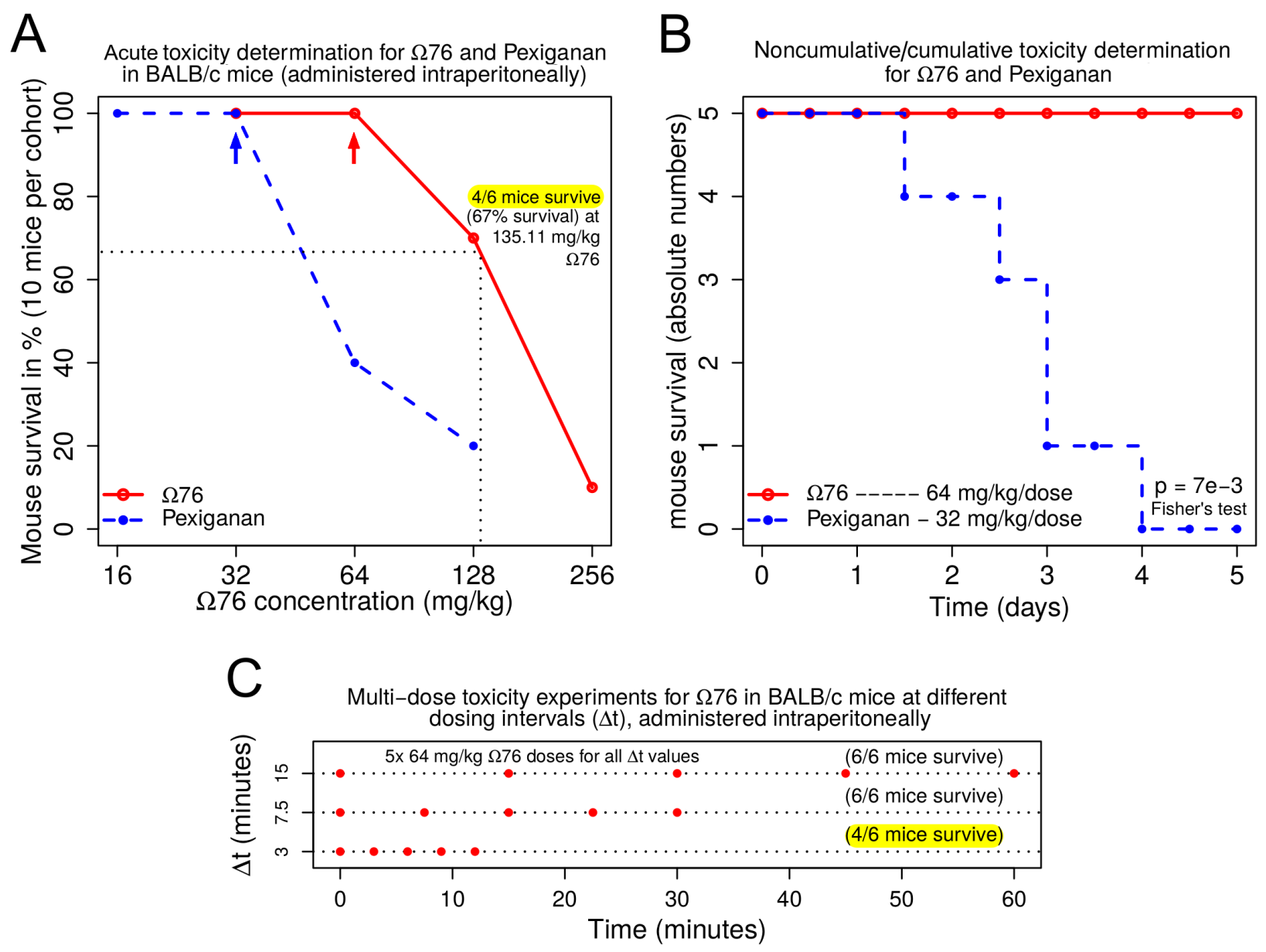

Figure 1. All experiments required to determine the elimination half-life using TOX $H_{L}$. BALB/c mice (6-8 weeks, $20 \mathrm{~g}$ ) were used for all experiments. (A) Acute toxicity experiments to determine survival vs. concentration of drug injected intraperitoneally. Determining the maximum nonlethal dose (MND), which is the highest concentration of a drug that causes no mortality, is essential for later noncumulative toxicity experiments. Here, the MND for Pexiganan is $32 \mathrm{mg} / \mathrm{kg}$ (blue arrow), and the MND for $\Omega 76$ is $64 \mathrm{mg} / \mathrm{kg}$ (red arrow). (B) Multi-dose (non)cumulative toxicity determination experiments. Here, mice were intraperitoneally injected with 11 doses of drug (at the MND concentration), at 12 hour intervals over the course of 5 days. All mice treated with Pexiganan died, indicating cumulative toxicity. All mice treated with $\Omega 76$ survived, indicating noncumulative toxicity. The difference between the survival outcomes of the $\Omega 76$ and Pexiganan cohorts are statistically significant ( $\mathrm{p}=7 \mathrm{e}-3$, Fisher's test). (C) After $\Omega 76$ displayed noncumulative toxicity, we determined the dosing interval $(\Delta \mathrm{t})$ between MNDs at which mortality is first observed. 5 doses at the MND were injected intraperitoneally at $\Delta \mathrm{t}$ of $15,7.5$ and 3 minutes. $67 \%$ survival (33\% mortality) was observed a $\Delta \mathrm{t}=3$ minutes $\left(\Delta \mathrm{t}_{M}\right.$, highlighted in yellow). The expected concentration of $\Omega 76$ responsible for $67 \%$ survival was calculated from panel $\mathbf{A}$ using linear interpolation (non-logarithmic), and found to be $135.11 \mathrm{mg} / \mathrm{kg}$. Note that a separate set of similar experiments was described in our previous work ${ }^{19}$, but these experiments were only used to assess the toxicity of $\Omega 76$. 
bioRxiv preprint doi: https://doi.org/10.1101/761585; this version posted September 8, 2019. The copyright holder for this preprint (which was not certified by peer review) is the author/funder, who has granted bioRxiv a license to display the preprint in perpetuity. It is made available under aCC-BY 4.0 International license.
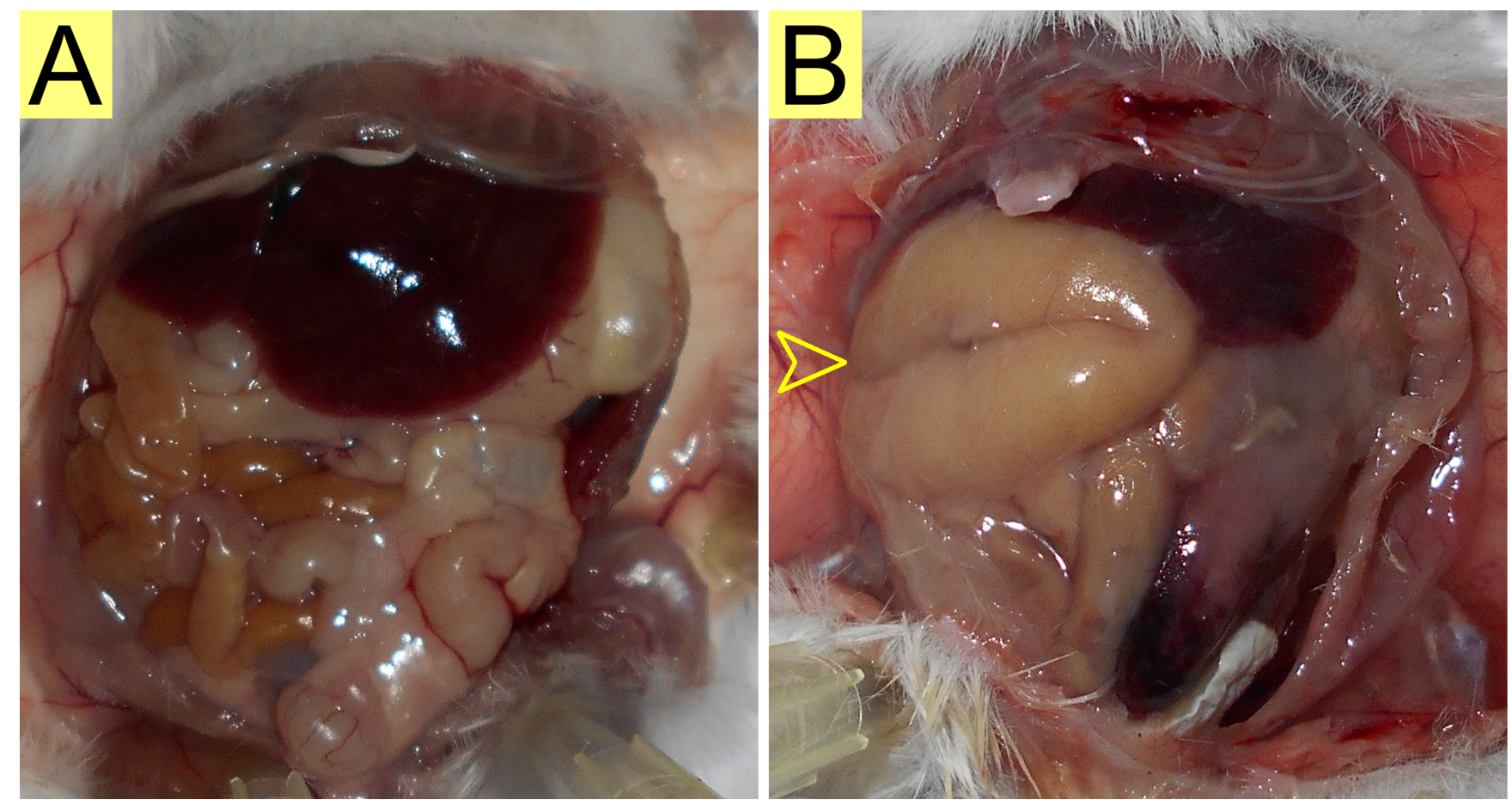

Figure 2. Peritonea of BALB/c mice (6-8 weeks, $20 \mathrm{~g}$ ) treated with $\Omega 76$ and Pexiganan for (non)cumulative toxicity experiments. (A) Peritoneum of a mouse treated with 11 doses of $64 \mathrm{mg} / \mathrm{kg} \Omega 76$ administered at 12 hour intervals over 5 days, and euthanized immediately after the last dose. All peritoneal organs appear intact. (B) Peritoneum of a mouse treated with 32 $\mathrm{mg} / \mathrm{kg}$ Pexiganan administered at 12 hour intervals until mortality occurred. Here, intestinal inflammation is clearly visible (yellow arrow) and may be the cause of cumulative toxicity. 
percentage survival of mice at $\Delta t_{M}$. We determined the $\Delta \mathrm{t}_{M}$ by intraperitoneally injecting BALB/c mice (6-8 weeks, $\left.20 \mathrm{~g}\right)$ with 5 doses of $\Omega 76$ at the MND $(64 \mathrm{mg} / \mathrm{kg})$ at different dosing intervals $(\Delta \mathrm{t}=15,7.5$, and 3 minutes, Figure 1C). All mice were observed for 5 days. All mice survived $\Omega 76$ treatment for 5 days at $\Delta t=15$ and 7.5 minutes. At $\Delta t=3$ minutes, only $4 / 6$ mice (67\%) survived for 5 days. Therefore, $\Delta \mathrm{t}_{M}=3$ minutes and $S_{\Delta t_{M}}=67 \%$. Note that unlike the $L D_{50}$ or MND, $\Delta \mathrm{t}_{M}$ is not a constant for a given drug. Mortality would also be expected at all $\Delta \mathrm{t} \leq 3$ minutes. However, the ratio of $S_{\Delta t_{M}}: \Delta \mathrm{t}_{M}$ is expected to remain proportional, provided $S_{\Delta t_{M}}>0 \%$. We recommend that a user must pick $\Delta \mathrm{t}$ values depending on the expected half life of the molecule being tested. If even the expected half life is unknown, a user can narrow down on the range using 2 -fold $\Delta t$ increases/decreases from an arbitrary starting $\Delta \mathrm{t}$.

\subsection{Using $\operatorname{TOX}_{H L}$ to calculate the elimination half-life}
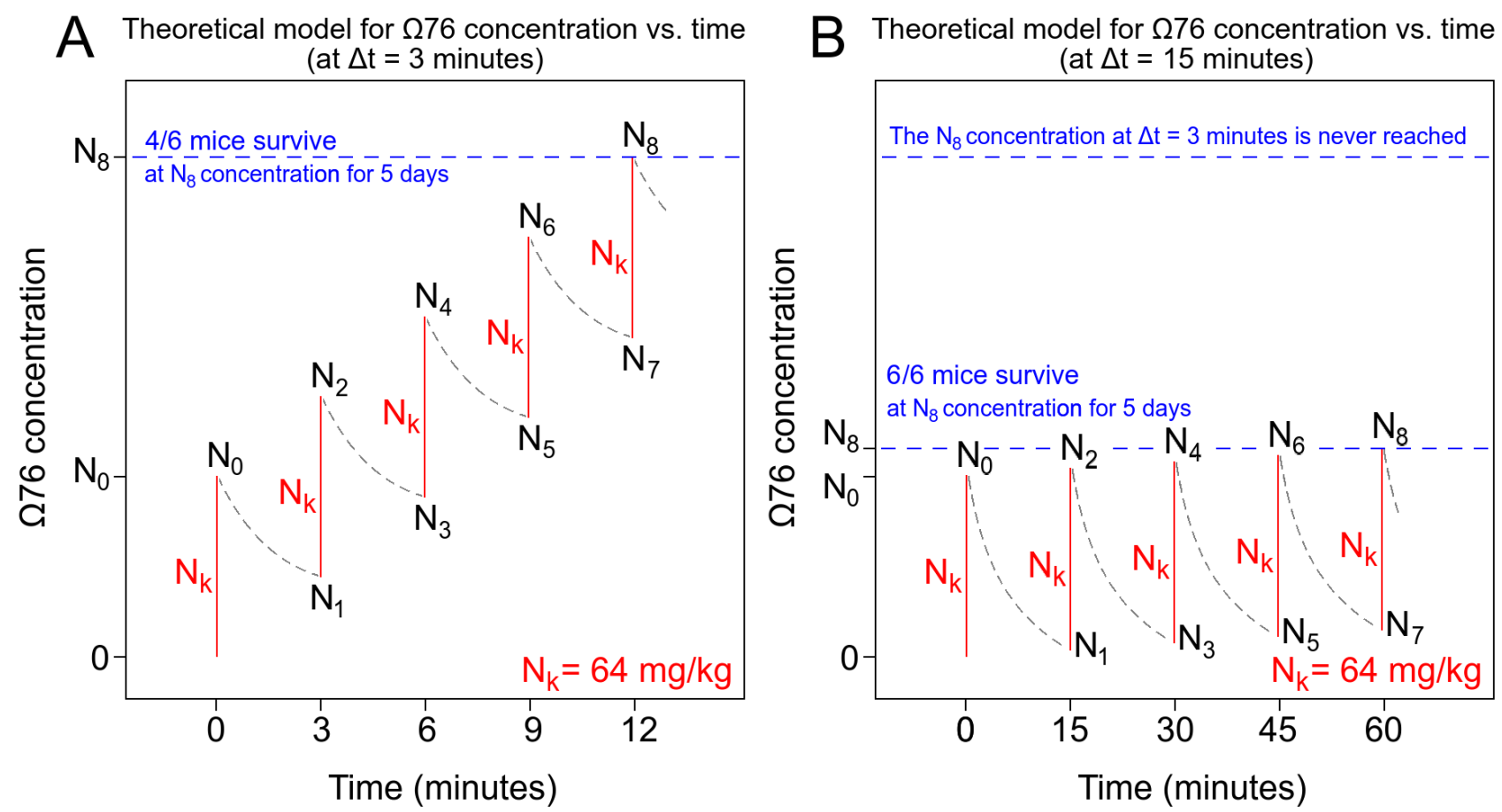

Figure 3. Theoretical pharmacokinetics of a multi-dose experiment involving administration of 5 doses of $\Omega 76$ at the MND $\left(64 \mathrm{mg} / \mathrm{kg}\right.$, here denoted as $N_{k}$ ). (A) At $\Delta \mathrm{t}=3$ minutes (previously described in Figure 1C). $\Omega 76$ absorption into the peritoneum is modeled as instantaneous (red lines), as intraperitoneal injections can be administered in 1-2 seconds. $\Omega 76$ elimination from the peritoneum is modeled using first order kinetics (exponential elimination, gray curves). From Figure 1A,C, we determined that the highest $\Omega 76$ concentration reached in the peritoneum $\left(N_{8}\right)$ was $135.11 \mathrm{mg} / \mathrm{kg}$. Given $\Delta \mathrm{t}, N_{k}$, and $N_{8}$, the values of $N_{0} \rightarrow N_{8}$, can easily be determined. These values would allow us to calculate the in vivo half-life of $\Omega 76$. (B) For contrast, the expected pharmacokinetics of $\Omega 76$ at $\Delta \mathrm{t}=15$ minutes is provided. Note that the concentration of $\Omega 76$ will not reach the previous $N_{8}$ concentration (at $\Delta \mathrm{t}=3$ minutes) after 5 doses, and no mortality will occur.

$\mathrm{TOX}_{H L}$ provides an estimate of in vivo half-life $\left(T_{1 / 2}\right)$ using only the values for $\mathrm{MND}, \Delta \mathrm{t}_{M}, S_{\Delta t_{M}}$, and the acute toxicity plot depicted in Figure 1A. TOX ${ }_{H L}$ begins by constructing a theoretical concentration vs. time model (Figure 3A) for the experiment used to determine $\Delta \mathrm{t}_{M}$ (Figure 1C). $5 \Omega 76$ doses at the MND $\left(64 \mathrm{mg} / \mathrm{kg}\right.$ ) were administered at $\Delta \mathrm{t}_{M}=3$ minutes. $\Omega 76$ absorption into the peritoneum is modeled as instantaneous, as peritoneal injections can be administered in 1-2 seconds. The peritoneal elimination of $\Omega 76$ is modeled as a first order (exponential) process. Over the course of 12 minutes $\left(4 \times \Delta \mathrm{t}_{M}\right)$, the $\Omega 76$ peritoneal concentration varies from 0 to $\mathrm{N}_{8}$, with intermediate concentrations ranging from $N_{1} \rightarrow N_{7}$. $N_{k}$ represents a constant concentration increase corresponding to the MND of $64 \mathrm{mg} / \mathrm{kg}$. For contrast, a concentration vs. time model for a larger dosing interval ( $\Delta t=15$ minutes) is also provided (Figure 3B). 
Figure 3 can also be described mathematically, using Equation-set 1:

$$
\begin{array}{rr}
N_{0}=N_{k} & N_{1}=N_{0} e^{-\lambda \Delta t_{M}} \\
N_{2}=N_{1}+N_{k} & N_{3}=N_{2} e^{-\lambda \Delta t_{M}} \\
N_{4}=N_{3}+N_{k} & N_{5}=N_{4} e^{-\lambda \Delta t_{M}} \\
N_{6}=N_{5}+N_{k} & N_{7}=N_{6} e^{-\lambda \Delta t_{M}} \\
& N_{8}=N_{7}+N_{k} \\
\text { Note }: & T_{1 / 2}=\frac{\log _{e} 2}{\lambda}
\end{array}
$$

Equation-set 1 can be simplified as described in Equation-set 2.

$$
\begin{array}{r}
N_{8}=N_{7}+N_{k} \\
N_{8}=N_{6} e^{-\lambda \Delta t_{M}}+N_{k} \\
N_{8}=\left(N_{5}+N_{k}\right) e^{-\lambda \Delta t_{M}}+N_{k} \\
\vdots(\text { expanding \& simplifying }) \\
\frac{N_{8}}{N_{k}}=e^{-4 \lambda \Delta t_{M}}+e^{-3 \lambda \Delta t_{M}}+e^{-2 \lambda \Delta t_{M}}+e^{-\lambda \Delta t_{M}}+1 \\
\text { Let } e^{-\lambda \Delta t_{M}}=x \\
\frac{N_{8}}{N_{k}}=x^{4}+x^{3}+x^{2}+x+1 \\
\vdots(\text { simplifying geometric series }) \\
\frac{N_{8}}{N_{k}}=\frac{1-x^{5}}{1-x}
\end{array}
$$

$S_{\Delta t_{M}}(67 \%)$ mice survive at concentration $\mathrm{N}_{8}$ (Figure 1C, highlighted). Using linear (non-logarithmic) interpolation, $S_{\Delta t_{M}}$ survival occurs at a single-dose $\Omega 76$ concentration of $135.11 \mathrm{mg} / \mathrm{kg}$ (Figure $1 \mathrm{~A}$, highlighted). Once the value of $\mathrm{N}_{8} / \mathrm{N}_{k}$ is known, Equation-set 2 can be solved for $x . T_{1 / 2}$ can be calculated from $x$ using Equation-set 3 .

$$
\begin{array}{r}
e^{-\lambda \Delta t_{M}}=x \\
\therefore \lambda=-\frac{\log _{e}(x)}{\Delta t_{M}} \\
T_{1 / 2}=\frac{\log _{e}(2)}{\lambda} \\
\therefore T_{1 / 2}=-\log _{e}(2) \times \frac{\Delta t_{M}}{\log _{e}(x)}
\end{array}
$$

For $\Omega 76, \mathrm{~N}_{8} / \mathrm{N}_{k}=135.11 / 64=2.11, x=0.55$, and $T_{1 / 2}=3.48$ minutes.

Note that the description of $\mathrm{TOX}_{H L}$ provided above describes equations for a 5-dose cumulative toxicity experiment (Figure 1C). The solution provided in Equation-set 2 can easily be modified for an $n$-dose cumulative toxicity experiment (Equation 4, where $n \geq 2$ ).

$$
\frac{N_{8}}{N_{k}}=\frac{1-x^{n}}{1-x}
$$


All experiments and calculations required to calculate $T_{1 / 2}$ using for $\mathrm{TOX}_{H L}$ are provided in Figure 4. A python script that calculates $T_{1 / 2}$ using TOX $_{H L}$ is provided on GitHub (https://github.com/preetham-V/TOX_HL) and is made available as a webserver (http://proline.biochem.iisc.ernet.in/toxhl/).

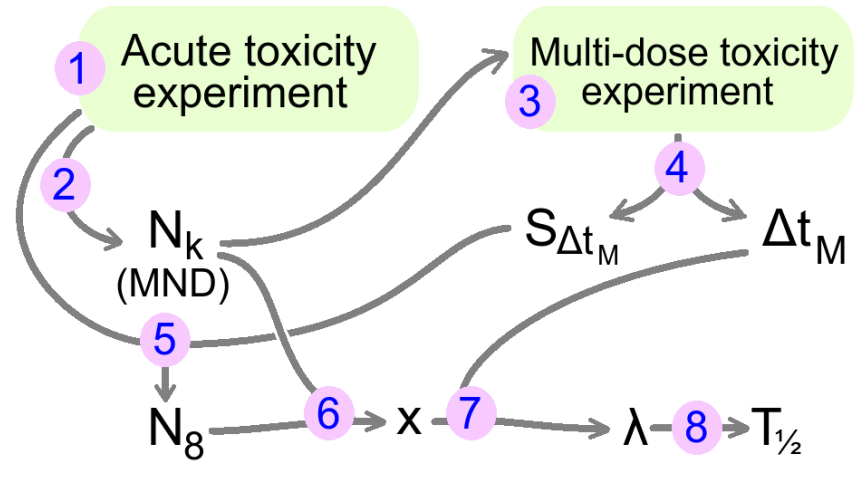

Figure 4. All experiments and calculations used by $\mathrm{TOX}_{H L}$ to calculate $T_{1 / 2}$ are provided in order. (1): Perform the acute toxicity experiment (Figure 1A). (2): obtain $N_{k}$ value from acute toxicity experiment. (3): Use $N_{k}$ to guide the dosing concentration for the multi-dose toxicity experiment (Figure 1B). (4): Obtain values for $\Delta t_{M}$ and $S_{\Delta t_{M}}$ from the multi-dose toxicity experiment. (5): Calculate $N_{8}$ concentration by looking at the mortality corresponding to $S_{\Delta t_{M}}$ in the acute toxicity graph. (6): Calculate $x$ using the values of $N_{k}$ and $N_{8}$, using Equation-set 2. (7,8): Use $x$ to calculate $\lambda$ and $T_{1 / 2}$ using Equation-set 3.

\subsection{Validating TOX $_{H L}$ using data from a standard pharmacokinetic assay}

Our previous work involved the pharmacokinetic characterization of $\Omega 76$ in mice ${ }^{19} .70 \mathrm{mg} / \mathrm{kg} \mathrm{N}$ selenomethionine-labeled $\Omega 76$ (Nselmet- $\Omega 76$, the molar equivalent of $64 \mathrm{mg} / \mathrm{kg}$ unlabeled $\Omega 76$ ) was injected intraperitoneally into BALB/c mice (6-8 weeks, $20 \mathrm{~g}$ ), and withdrawn at different time intervals using cardiac punctures. We obseved that Nselmet- $\Omega 76$ reached a serum concentration maxima $\left(C_{\max }\right)$ at 4.59 minutes post-injection. This indicates that the peritoneal elimination half-life of $\Omega 76$ is within the range of $0 \rightarrow 4.59$ minutes, and is in good agreement with the $\mathrm{TOX}_{H L}$ value of 3.48 minutes.

\section{Discussion}

In this work, we described $\operatorname{TOX}_{H L}$, using which the half-life of a drug can be determined based on the survival outcomes of 3 simple experiments performed on mice. $\mathrm{TOX}_{H L}$ is cost-effective, easy to perform, and requires no instruments or special reagents. We described and validated $\mathrm{TOX}_{H L}$ using an example antimicrobial peptide $(\Omega 76)$. Using $\mathrm{TOX}_{H L}$, the peritoneal half-life of $\Omega 76$ in mice was found to be 3.48 minutes. This value was in good agreement with the peritoneal half-life range of $0 \rightarrow 4.59$ minutes, calculated using a conventional ICP-MS technique that tracked selenium. A python script (https: // github.com/preetham-V/TOX_HL) and webserver(http://proline.biochem.iisc.ernet.in/toxhl/) implementing $\mathrm{TOX}_{H L}$ have beem made available to the community.

$\mathrm{TOX}_{H L}$ can be modified in many ways to meet a user's requirements. We have used 5 intraperitoneal doses $(n)$ to determine ${ }_{M}$ and $S_{M}$ (Figure 1C). However, any number of doses $(2 \leq n<\infty)$ may be used. $S_{M}$ will increase with $n$, which is advantageous as it eliminates time-errors when dosing at a small . However, the amount of drug used will increase with $n$, necessitating the synthesis/purchase of greater quantities. The total volume of drug injected intraperitoneally will also increase. Extreme values of $n$ (for example, 20 doses of $200 \mu \mathrm{L}$ each), would cause abdominal distention in mice, altering the elimination kinetics. Larger $n$ should therefore be accompanied by smaller injection volumes. TOX $_{H L}$ models first order elimination kinetics (3). For exceptional drugs following non-exponential kinetics, a user can replace the exponential equations in Equation-set 1 with decay equations of their choosing. TOX ${ }_{H L}$ can also be used to calculate the bloodstream half-life of drugs, simply by performing intravenous rather than intraperitoneal injections in mice.

It should be noted that $\mathrm{TOX}_{H L}$ was only validated using a single molecule $(\Omega 76)$. Additional experiments using more molecules would help further validate our approach. $\mathrm{TOX}_{H L}$ can only be used for drugs possessing noncumulative toxicity, which has to 
be tested prior to half-life calculation (Figure 1B). TOX ${ }_{H L}$ requires a larger number of mice in comparison to conventional methods. Consequently, a larger amount of drug is required for these tests.

Nevertheless, we expect $\mathrm{TOX}_{H L}$ to be especially useful for the half-life determination of a library of compounds, created from a parent compound displaying noncumulative toxicity. For example, a 100-1000 member peptide library, created using saturation mutagenesis of a parent antimicrobial peptide, could quickly and easily be assayed using $\operatorname{TOX}_{H L}$.

\section{Methods}

For all experiments described in this work, BALB/c mice (6-8 weeks, $20 \mathrm{~g}$ ) were housed at the Central Animal Facility (CAF, IISc) and fed ad libitum with pellet feed and water. All animal experiments were approved by the Institutional Animal Ethics Committee, IISc (Project No. CAF/Ethics/550/2017). A detailed description of all methods used is provided in the results section. However, it should be noted that $20 \mathrm{mg} / \mathrm{mL}$ stocks for both $\Omega 76$ and Pexiganan were prepared in physiological saline $(0.8 \% \mathrm{NaCl})$ and stored at $-80{ }^{\circ} \mathrm{C}$. Both peptides display poorer solubility in phosphate buffered saline (PBS). These stocks were diluted in saline to obtain the concentration needed for the specific experiment. All intraperitoneal injections were $200 \mu \mathrm{L}$ in volume, as larger volumes could alter peritoneal elimination kinetics.

\section{References}

1. McNaught, A. D. \& McNaught, A. D. Compendium of chemical terminology, vol. 1669 (Blackwell Science Oxford, 1997).

2. Taft, D. R. Drug excretion. In Pharmacology, 175-199 (Elsevier, 2009).

3. Rollins, D. E. \& Klaassen, C. D. Biliary excretion of drugs in man. Clin. pharmacokinetics 4, 368-379 (1979).

4. Mathur, D. et al. Peplife: a repository of the half-life of peptides. Sci. reports 6, 36617 (2016).

5. Gorodischer, R. \& Koren, G. Salivary excretion of drugs in children: theoretical and practical issues in therapeutic drug monitoring. Dev. pharmacology therapeutics 19, 161-177 (1992).

6. Ito, S. \& Lee, A. Drug excretion into breast milk—overview. Adv. drug delivery reviews 55, 617-627 (2003).

7. Crandall, D. C. \& Leopold, I. H. The influence of systemic drugs on tear constituents. Ophthalmology 86, 115-125 (1979).

8. Chandrasekaran, S., Bayne, W. \& Shaw, J. E. Pharmacokinetics of drug permeation through human skin. J. pharmaceutical sciences 67, 1370-1374 (1978).

9. Cone, E. J. Mechanisms of drug incorporation into hair. Ther. drug monitoring 18, 438-443 (1996).

10. Andrew, B. \& Shargel, L. Applied Biopharmaceutics \& Pharmacokinetics (Mcgraw-hill Education-Europe, 2015).

11. Kauffman, R. E. et al. Pharmacokinetics of chloramphenicol and chloramphenicol succinate in infants and children. The J. pediatrics 98, 315-320 (1981).

12. Li, J. et al. Pharmacokinetics of colistin methanesulphonate and colistin in rats following an intravenous dose of colistin methanesulphonate. J. Antimicrob. Chemother. 53, 837-840 (2004).

13. Formelli, F., Pollini, C., Casazza, A. M., di Marco, A. \& Mariani, A. Fluorescence assays and pharmacokinetic studies of 4-deoxydoxorubicin and doxorubicin in organs of mice bearing solid tumors. Cancer chemotherapy pharmacology $\mathbf{5}$, 139-144 (1981).

14. Alberts, D. S., Bachur, N. R. \& Holtzman, J. L. The pharmacokinetics of daunomycin in man. Clin. Pharmacol. \& Ther. 12, 96-104 (1971).

15. Heeg, J. F., Hiser, M. F., Satonin, D. K. \& Rose, J. Q. Pharmacokinetics of probucol in male rats. J. pharmaceutical sciences 73, 1758-1763 (1984).

16. Paxton, J., Rowell, F. \& Cree, G. Comparison of three radioligands, selenium-75, lodine-125, and tritium, in the radioimmunoassay of methotrexate. Clin. chemistry 24, 1534-1538 (1978).

17. Back, D. et al. The effects of ampicillin oral contraceptive steroids in women. Br. journal clinical pharmacology 14, 43-48 (1982).

18. Michiels, M., Hendriks, R. \& Heykants, J. A sensitive radioimmunoassay for fentanyl. Eur. J. Clin. Pharmacol. 12, 153-158 (1977). 
19. Nagarajan, D. et al. $\omega 76$ : A designed antimicrobial peptide to combat carbapenem-and tigecycline-resistant acinetobacter baumannii. Sci. advances 5, eaax1946 (2019).

20. Ge, Y. et al. In vitro antibacterial properties of pexiganan, an analog of magainin. Antimicrob. agents chemotherapy 43, 782-788 (1999).

21. Lamb, H. M. \& Wiseman, L. R. Pexiganan acetate. Drugs 56, 1047-1052 (1998).

\section{Author contributions statement}

D.N. conceived and designed $\mathrm{TOX}_{H L}$, and performed all experiments. P.V. created a python script and webserver implementing TOX $_{H L}$. C.T. and A.D. co-performed mouse experiments. D.C. and N.C. coordinated the study, planned experiments, and provided resources. All authors reviewed the final draft of the manuscript. 